\title{
Index of Refraction Sensors: Virtually Unlimited Sensing Power at Critical Angle
}

\author{
Ruggero Micheletto and Ishii Hikaru \\ Yokohama City University, 22-2 Seto, Kanagawa-ku, Yokohama, 236-0026 Japan
}

\begin{abstract}
We demonstrate analytically that discontinuity at critical angle can be used to reach extremely high sensitivities against any optical properties that modify this angular value. To test in practice the approach we fabricated cheap and basic sensors by which we could demonstrate extremely high index of refraction discrimination ability in the range of one part over a million. Moreover, we constructed a similar apparatus to detect in a very simple and low-cost manner nanometer scale mechanical vibrations. Also in this case we obtained very high sensitivity, tiny vibrations of $2.4 \mathrm{~nm}$ were resolved, this correspond to an angular shift of about $50 * 10^{-6} \mathrm{deg}$, as far as we know the best angular sensitivity recorded so far. The critical angle detection method discussed in this paper is applicable generally to any optical system and may pave the way for next generation optical sensing devices.
\end{abstract}

(C) 2010 Optical Society of America

OCIS codes: $120.0280,120.4530,240.6680,120.7280,280.4788$

The recent need of integration and miniaturization has motivated the scientific community to develop smaller and more efficient optical detectors. This paper discuss of a simple optical principle applied to realize very high sensitivity sensors. In particular, we focused on showing that such systems would have virtually infinite sensitivity and that performances, in case of pure index of refraction sensing, would be better than Surface Plasmon Resonance sensors (SPR) [1].
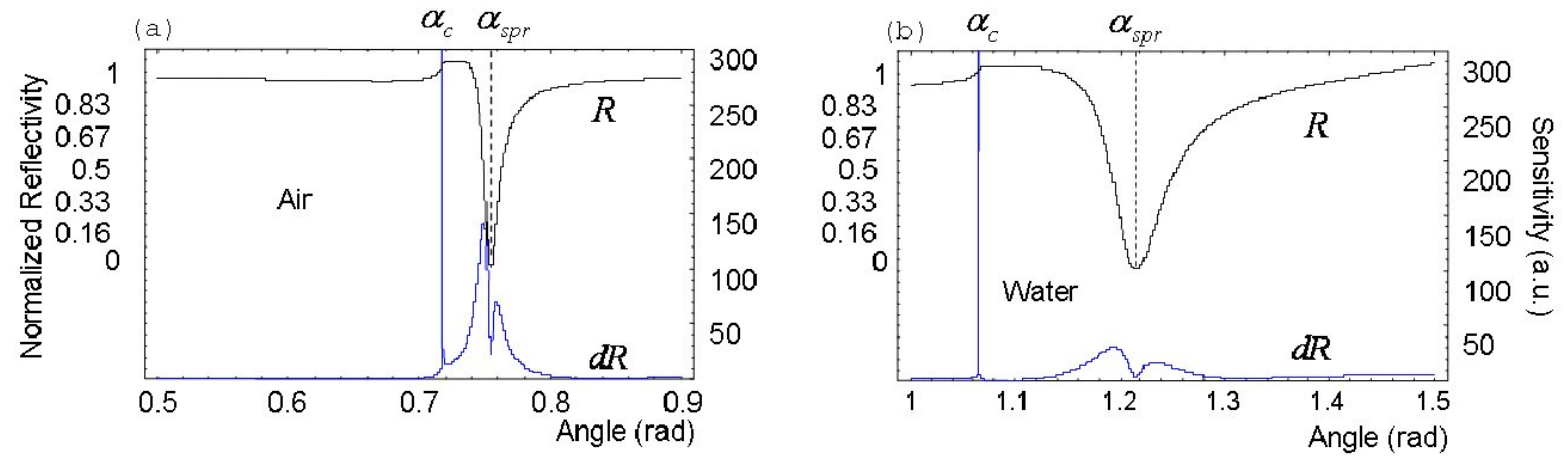

Fig. 1. Sensitivity curve for a Kretschmann three layer system. Calculations are including Surface Plasmon Resonance effect for comparison between critical angle and SPR sensitivity peaks. $R$ is the Reflectivity in function of angle $\alpha(\mathrm{rad})$. The SPR dip is visible at $\alpha_{s p r}$, together with a small shoulder at critical angle $\alpha_{c}$. The curve $d R$ is the numerical derivative of $R$, a very sharp and narrow peak is evident at critical angle. These plots are obtained from a three layer Fresnel planar model under illumination of $\lambda=670 \mathrm{~nm}$. Materials are glass $\left(n_{p}=1.52\right)$, a Gold $\left(\omega_{p}=1.3610^{16}, \omega_{t}=\right.$ $\left.1.4510^{14}, \varepsilon_{i}=9.75\right)$ layer $d=52 \mathrm{~nm}$ thick and water $\left(n_{s}=1.33\right)$ or air $\left(n_{s}=1\right)$ as sample materials. The SPR peak deteriorates to increased index of refraction, whereas sensitivity peak at $\alpha_{c}$ appears extremely sharp and unchanged.

To test the theoretical analysis shown in fig. 1, we realized a planar multireflection device, and with that we performed index of refraction measurements. The resulting sensing on Methanol and Water is shown in fig. 2A against 

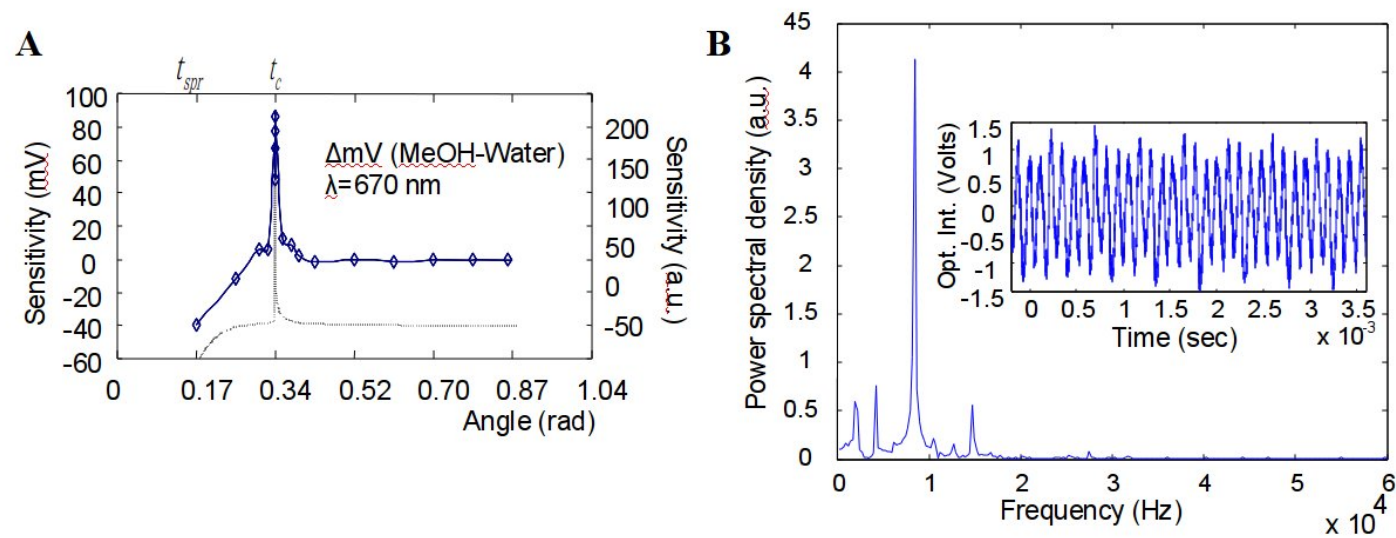

Fig. 2. Panel A: Test on sensitivity measured as response difference between water and methanol. Sharp sensitivity peak at critical angle is evident, theoretical curve is also shown. Panel B: The power spectrum of a calibrated $240 \mathrm{~nm}, 8 \mathrm{KHz}$ vibration applied to the sensor, the inset shows the signal detected at the optical detector.

the theoretical behavior. The discontinuity discussed reveals as a sharp peak at about $t_{c}=0.35$ rad in the actual experimental apparatus $\left(t=\pi / 4-\sin ^{-1}\left(n_{1} / n_{0} \sin (\alpha-\pi / 4)\right)\right)$, that corresponds to the critical angle $\alpha_{c}$ of a Kretschmann setup [2].

In an additional initial test with similar planar multireflection setup, we thought that we can exploit the same principle for a super-high sensitive vibration sensor. The optical system is setup in a way to respond to beam angular variation. So if we apply a tiny shock to the setup resulting in a very small angular displacement of the beam, we realize a very simple vibration sensor. To have a precise evaluation of the actual sensitivity to the angular displacements, we applied a controlled vibration signal using a thin calibrated piezo actuator. Given that we drive the actuator with 8 $\mathrm{KHz}$ sinusoidal signal of 12 volts peak to peak, waveguide is forced to oscillate in the $\mathrm{z}$ direction for a range of about $240 \mathrm{~nm}$. The highest angle deviation due to this vibration is in the order of $\delta \omega=20 * 10^{-6} \mathrm{rad}$. Even with these tiny angular displacement we could observe a detectable signal, figure 2B. More extreme calibrated tests demonstrated minimal vibration discrimination down to 2.4 nanometer peak-to-peak vibration at $8 \mathrm{KHz}$ [3].

We presented a new simple approach to index of refraction sensing, we proved analytically that sensitivity is unlimited and we tested experimentally the promising behavior reaching index of refraction discrimination in the order of $10^{-6}$ with a simple home made planar detector. While detection of local plasma perturbation in the vicinity of metal coating is reserved to the sole SPR operation mode, we demonstrated that sensitivity to the index of refraction is higher at $\alpha_{c}$. Moreover, we have shown that the concept of critical angle sensing can be extended to other kind of sensors, presenting a simple vibration sensor with excellen performances based on the same principle. Critical angle sensing realize a virtually unbounded sensitivity value, so instrumental constraints are the only limitation to various possible devices. We believe this approach can be useful to realize extremely sensitive systems for next generation detectors.

\section{References}

[1] R. Micheletto, K. Hamamoto, T. Fujii and Y. Kawakami, "Tenfold improved sensitivity using high refractive-index substrates for surface plasmon sensing", Applied Physics Letters, 93, 174104 (2008)

[2] R. Micheletto, K. Hamamoto, S. Kawai and Y. Kawakami, "Index-of-refraction sensors: virtually unlimited sensing power at critical angle", Optics Letters, 31, 2, 205-207 (2006)

[3] R. Micheletto, K. Hamamoto and Y. Kawakami, "Optical nanometer-scale sensing of mechanical vibrations with a planar glass at critical angle", Applied Physics Letters, 90, 244108 (2007) 